\title{
23. ON A POSSIBLE CORRUGATION \\ OF THE GALACTIC PLANE
}

\author{
C. M. VARSAVSKY and R. J. QUIROGA
}

Instituto Argentino de Radioastronomía, Buenos Aires, Argentine

\begin{abstract}
We have studied the rotation curve of the Galaxy at different heights below and above the equator. In the course of this work we noticed that the maximum brightness temperature of hydrogen oscillates around the galactic plane following a fairly sinusoidal pattern. It is further noticed that the maximum temperature of hydrogen occurs right on the plane in the regions where the rotation curve has a form indicating solid body rotation. A rotation curve based on poinis of maximum hydrogen temperature does not differ appreciably from a rotation curve measured on the galactic plane.
\end{abstract}

When the 30-meter telescope of the I.A.R.-C.I.W. Radio Astronomy Station was put into operation in June, 1966, one of its first tasks was to check the rotation curve of the Galaxy that had been measured previously at Parkes (Bajaja et al., 1967). Further work on the rotation curve involved measuring the curve at heights of 50 and 100 parsecs above, and 100 parsecs below, the galactic plane. The results, shown in Figure 1, indicate that at $z=50 \mathrm{pc}$ the rotation curve obtained is practically identical with that at $z=0$, while the curves at $z= \pm 100 \mathrm{pc}$ indicate slower rotation.

To carry out this work, observations were made at $1^{\circ}$ intervals in longitude and, at each longitude, the latitude was chosen so that the tangential point would be at the proper height. Comparison of the observations at different latitudes gave indication that the maximum brightness temperature at the tangential point was not normally at $b^{\mathrm{II}}=0^{\circ}$ but rather at some height below or above the equator.

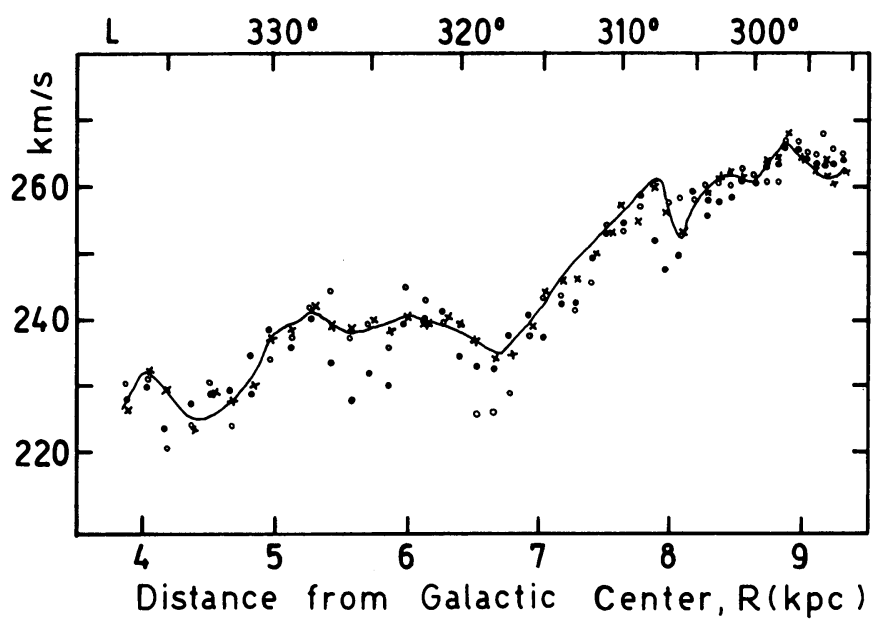

Fig. 1. The rotation curve of the Galaxy at different heights. The full line shows the curve for $z=0$. Open circles correspond to $z=100 \mathrm{pc}$; filled circles to $z=-100 \mathrm{pc}$; crosses to $z=50 \mathrm{pc}$. 

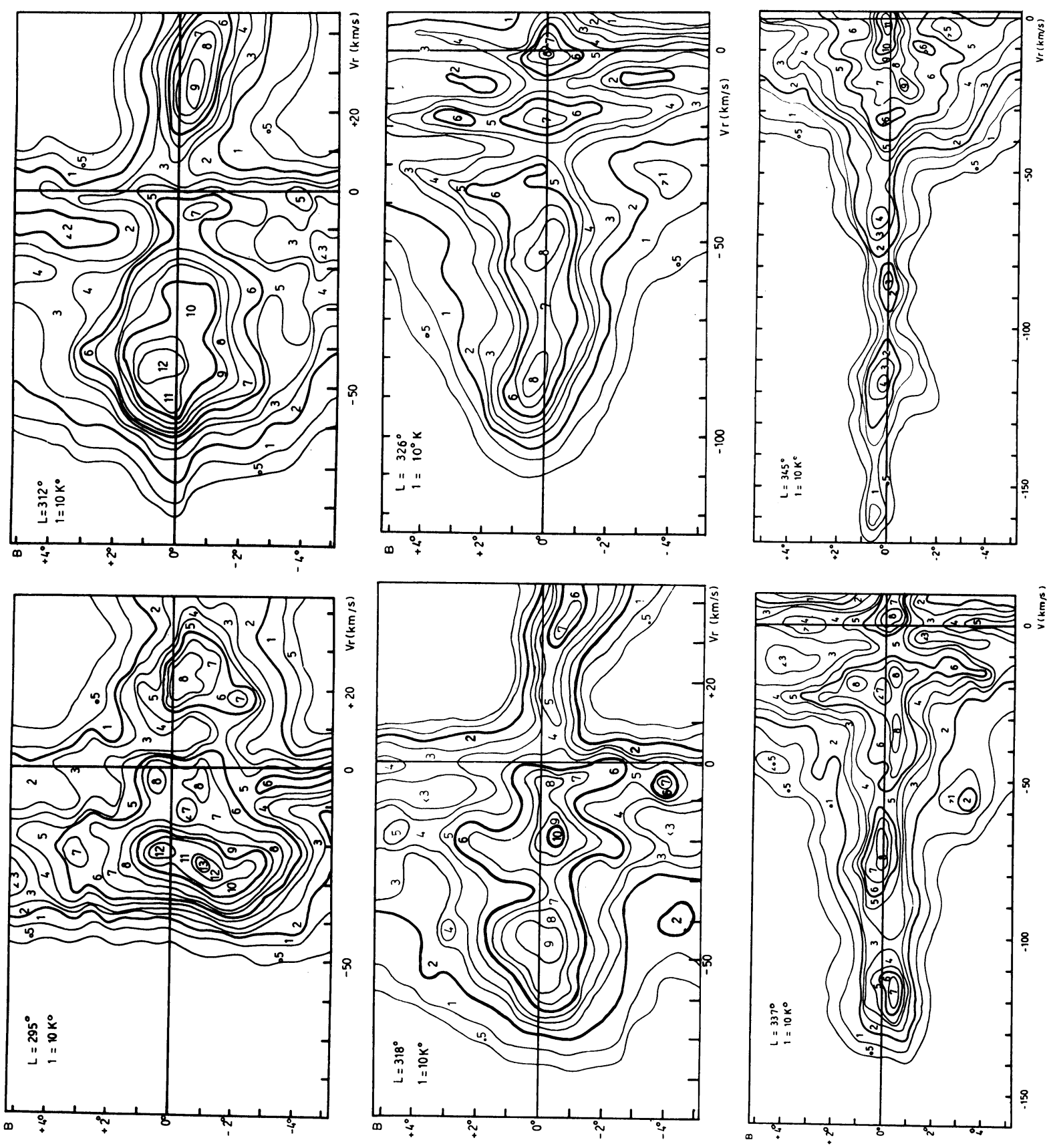
To study this effect in detail, as well as other properties of the hydrogen near the galactic plane that will be considered in a later publication, we carried out a survey in the region $281^{\circ} \leqslant l^{\mathrm{II}} \leqslant 345^{\circ}$ and $-5^{\circ} \leqslant b^{\mathrm{II}} \leqslant 5^{\circ}$, with spacings of $2^{\circ}$ or $3^{\circ}$ in $l^{\mathrm{II}}$ and 0.5 in $b^{\mathrm{II}}$. From the profiles we prepared contour diagrams of brightness temperature as a function of galactic latitude and radial velocity, for given longitudes. Samples of such contour diagrams are shown in Figure 2.

As can be seen easily from Figure 2, the points of highest brightness temperature with maximum radial velocity, that is, at the tangential point, occur at different heights below or above the equator. Figure 3 shows this effect in detail.



Fig. 3. Height dependence of the points of maximum brightness temperature as a function of distance to the galactic center.

The curve in Figure 3 presents several interesting features. In the first place, the general shape of the curve is sinusoidal, and indicates that the galactic plane is 'corrugated', with a wavelength of about $3 \mathrm{kpc}$. Secondly, it is quite remarkable that the two regions, centered around 4.6 and $7.3 \mathrm{kpc}$ from the center of the Galaxy, where the greatest density of hydrogen seems to be exactly on the equator, correspond to the portions of the rotation curve where the velocity varies linearly with distance, indicating solid body rotation. Thirdly, we see that for $8.4 \leqslant R \leqslant 9.0 \mathrm{kpc}$ there are two concentrations of hydrogen, one below and one above the galactic plane, with the same radial velocity.

Finally, Figure 4 shows a comparison of the rotation curve obtained at the plane with one using the points that have the highest brightness temperature. We see that both curves are essentially identical; in the second curve the dip at $R=8.1 \mathrm{kpc}$

Fig. 2a-f. Contour diagrams of brightness temperature as a function of galactic latitude and radial velocity for different longitudes. 


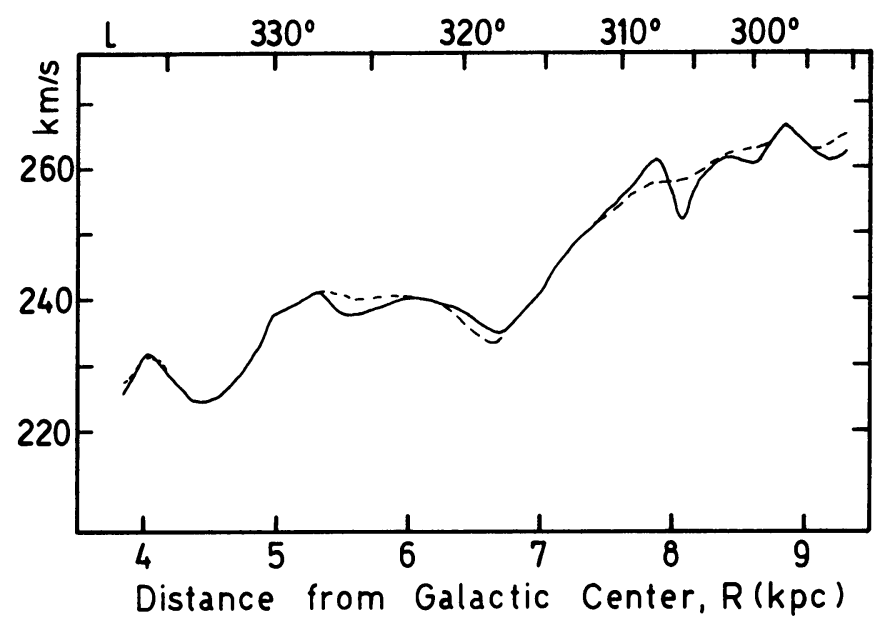

Fig. 4. The rotation curve of the galaxy at $b^{\mathrm{II}}=0$ (full line) and at the heights of maximum brightness temperature (dotted line).

disappears, and a few other points are shifted to slightly higher velocities, but not nearly enough to make the curve obtained for the fourth quadrant coincide with that obtained by Kerr or by Shane and Bieger-Smith (1966) for the first quadrant.

\section{Acknowledgement}

One of us (R. J. Q.) wishes to thank the Consejo Nacional de Investigaciones Científicas y Técnicas of Argentina for a scholarship that made this work possible.

\section{References}

Bajaja, E., Garzoli, S., Strauss, F., and Varsavsky, C. M.: 1967, IAU Symposium No. 31, p. 181.

Shane, N. W. and Bieger-Smith, G. P.: 1966, Bull. Astron. Inst. Netherl. 18, 263. 